\title{
Agrilus (Pinarinus) dingoides n. sp. du nord de l'Australie (Coleoptera, Buprestidae)
}

\author{
par Gianfranco CURLETTI* \& Henri-Pierre ABERLENC** \\ *Museo Civico di Storia Naturale, Parco Cascina Vigna, Via S. Fr. di Sales 188, \\ I - 10022 Carmagnola, TO, Italie <gianfranco.curletti@yahoo.it> \\ **Cirad Bios, UMR CBGP (Inra/IRD/Cirad/Montpellier SupAgro), TA A-55/L, Campus de Baillarguet, \\ F-34398, Montpellier cedex 5 <henri-pierre.aberlenc@cirad.fr>
}

Résumé. - Agrilus (Pinarinus) dingoides n. sp. est décrit du Territoire du Nord (Australie). Cette nouvelle espèce est comparée avec sa très proche voisine A. (Pinarinus) dingo Curletti, 2001.

Summary. - Agrilus (Pinarinus) dingoides n. sp. from Northern Australia (Coleoptera, Buprestidae). Agrilus (Pinarinus) dingoides n. sp. from Northern Territory (Australia) is described. This new species is compared with the close A. (Pinarinus) dingo Curletti, 2001.

Riassunto. - Agrilus (Pinarinus) dingoides n. sp. dell'Australia settentrionale (Coleoptera, Buprestidae). E'descritto Agrilus (Pinarinus) dingoides n. sp. del Northern Territory, morfologicamente affine ad $A$. (Pinarinus) dingo Curletti, 2001, con cui viene messo a confronto.

Keywords. - Australia, Northern Territory, biodiversity, taxonomy, Buprestidae, new species.

\section{Agrilus (Pinarinus) dingoides n. sp. (fig. 1)}

Holotype: §̃, Australia, NT, Kakadu NP, Jim Jim Billabong, 1256'S - 132 $33^{\circ} \mathrm{E}$, 5 m, 5-8.XII.2008, Sv. Bílý leg., in Museum of Northern Territory, Darwin (MNTD).

PARATYPE : $1 \hat{\jmath}$, idem holotype, in National History Museum, Praha (NMPC).

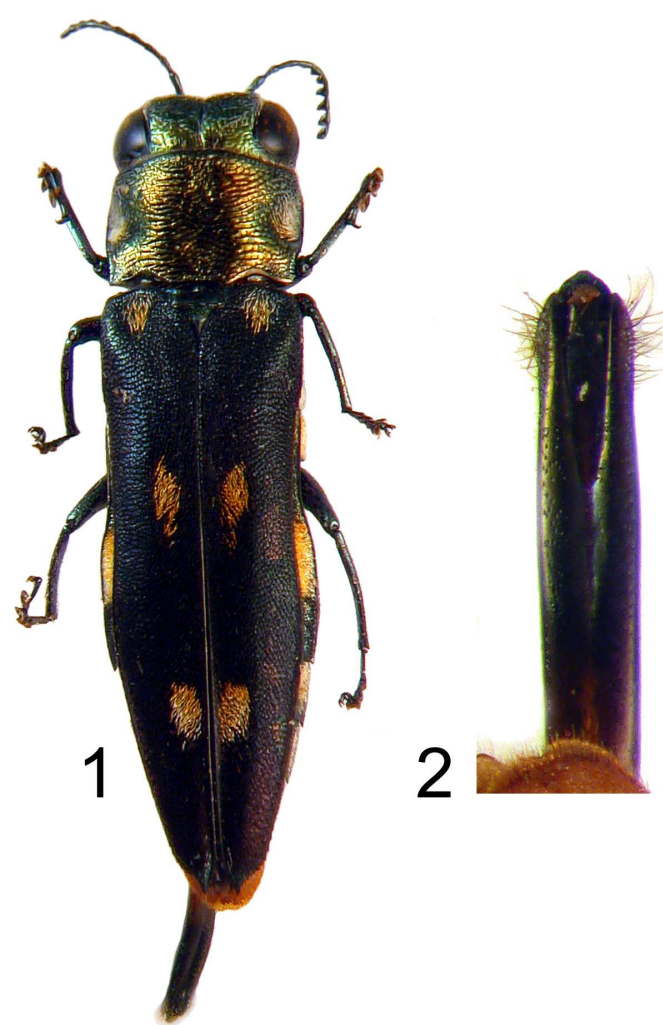

Fig. 1-2. - Agrilus (Pinarinus) dingoides n. sp. - 1, Holotype en vue dorsale. 2, Edéage en vue dorsale.
Description de l'holotype. - Longueur : 7,8 mm.

Tête. Tête sillonnée longitudinalement au milieu. Vertex large entre les yeux, d'une largeur plus grande que la moitié de celle de la marge antérieure du pronotum. Front glabre, plus large dans la partie supérieure. Yeux grands et saillants. Clypéus légèrement en relief par rapport à la base du front, dont il est séparé par une robuste carène transversale.

Thorax. Pronotum transverse, déprimé sur les côtés de la partie médiane, les marges latérales parallèles dans la moitié antérieure et sinuées dans la moitié postérieure, avec les angles basaux quelque peu obtus. Disque avec une forte sculpture composée de rides approximativement transversales. Carinule préhumérale peu visible, entière, brève, confluente avec la carène marginale dans la moitié basale. Carènes marginales confluentes, soudées à la base, avec la submarginale effacée dans la moitié antérieure. Mentonnière avec la marge antérieure non sinuée. Marges latérales de la plaque prosternale parallèles. Scutellum caréné transversalement. Apex élytral avec un mucron long et épais. Griffes antérieures bifides, les médianes et les postérieures dentées. Métatarse à peine plus court que le métatibia, avec le métatarsomère basal plus long que la somme des trois suivants $(1>2+3+4)$.

Abdomen. Dernier sternite visible avec la marge apicale arrondie. 
Coloration et pilosité. Tête verte, antennes bronzées. Pronotum doré, disque avec une tache de pubescence blanche pruineuse dans la partie latéro-antérieure. Scutellum noir. Elytres noirs, avec trois paires de taches de pubescence pruineuse jaune le long de la suture, respectivement au calus huméral, à $1 / 3$ et à $2 / 3$ de la longueur. Latérotergites avec une tache de pubescence basale. Plaque prosternale avec à la base un toupet de longs poils blancs. Pattes noires. Côtés de la face ventrale noirs, avec une pubescence jaune clair sur les métépisternes, les métacoxas et à la base des trois derniers sternites abdominaux.

Edéage. Longueur : 1,7 mm. Allongé, étroit, les paramères subparallèles (fig. 2).

Description du paratype. - Longueur : 7,5 mm. Nous n'avons observé aucune différence substantielle, sauf la taille et la coloration de la pubescence de la macule basale de l'élytre, qui est blanche.

Note comparative. - Les caractères morphologiques d'A. dingoides $\mathrm{n}$. sp. incitent à le classer dans le sous-genre Pinarinus (CURLETTI, 2001). A. dingoides n. sp. pourrait même être confondu avec A. dingo Curletti, 2001, déjà décrit du Northern Territory, mais il en diffère par quelques caractères importants présentés dans le tableau I.

Tableau I. - Caractères distinguant Agrilus dingo Curletti, 2001, de A. dingoides n. sp.

\begin{tabular}{|l|l|}
\hline \multicolumn{1}{|c|}{ A. dingo } & \multicolumn{1}{c|}{ A. dingoides } \\
\hline - plus grand $(>9,5 \mathrm{~mm})$ & - plus petit $(<8 \mathrm{~mm})$ \\
- pronotum vert & - pronotum doré \\
- carinule préhumérale non entière & - carinule préhumérale entière \\
- sclérite sous-gulaire sinué & - sclérite sous-gulaire non sinué \\
- mucron élytral moins prononcé & - mucron élytral plus allongé \\
\hline
\end{tabular}

Biologie. - La plante-hôte est inconnue. Les deux exemplaires, attirés par la lumière, ont été capturés au cours de deux nuits différentes (Bílý, comm. pers.). L'activité nocturne et un relatif phototropisme positif sont un cas exceptionnel et même extraordinaire chez les Buprestides en général et chez les Agrilus en particulier. L'un de nous (HPA) avait cependant déjà bioté à Vanikoro (îles Salomon) 27 exemplaires d'Agrilus (Agrilus) vestitus Deyrolle, 1864, au piège lumineux, et une centaine d'exemplaires aux pièges jaunes englués (BílÝ et al., 2006).

REMERCIEMENTS. - Nous remercions notre excellent collègue le Dr S. Bílý, de Prague, pour nous avoir donné l'opportunité d'étudier ces exemplaires.

\section{AUTEURS CITÉS}

Bílý S., Curletti G. \& ABerlenc H.-P., 2006. - Entomofauna of Vanikoro (Solomon Islands). Part

1. Introduction and Coleoptera: Buprestidae. Folia Heyrovskyana, A, 13 (4) (2005) : 163-172.

Curletti G., 2001. - The genus Agrilus in Australia. Jewel Beetles, 9 : 1-76. 